\title{
Estrategias didácticas para el fortalecimiento de la competencia de comunicación escrita en estudiantes universitarios
}

Teaching strategies for strengthening the written communication competence in university students

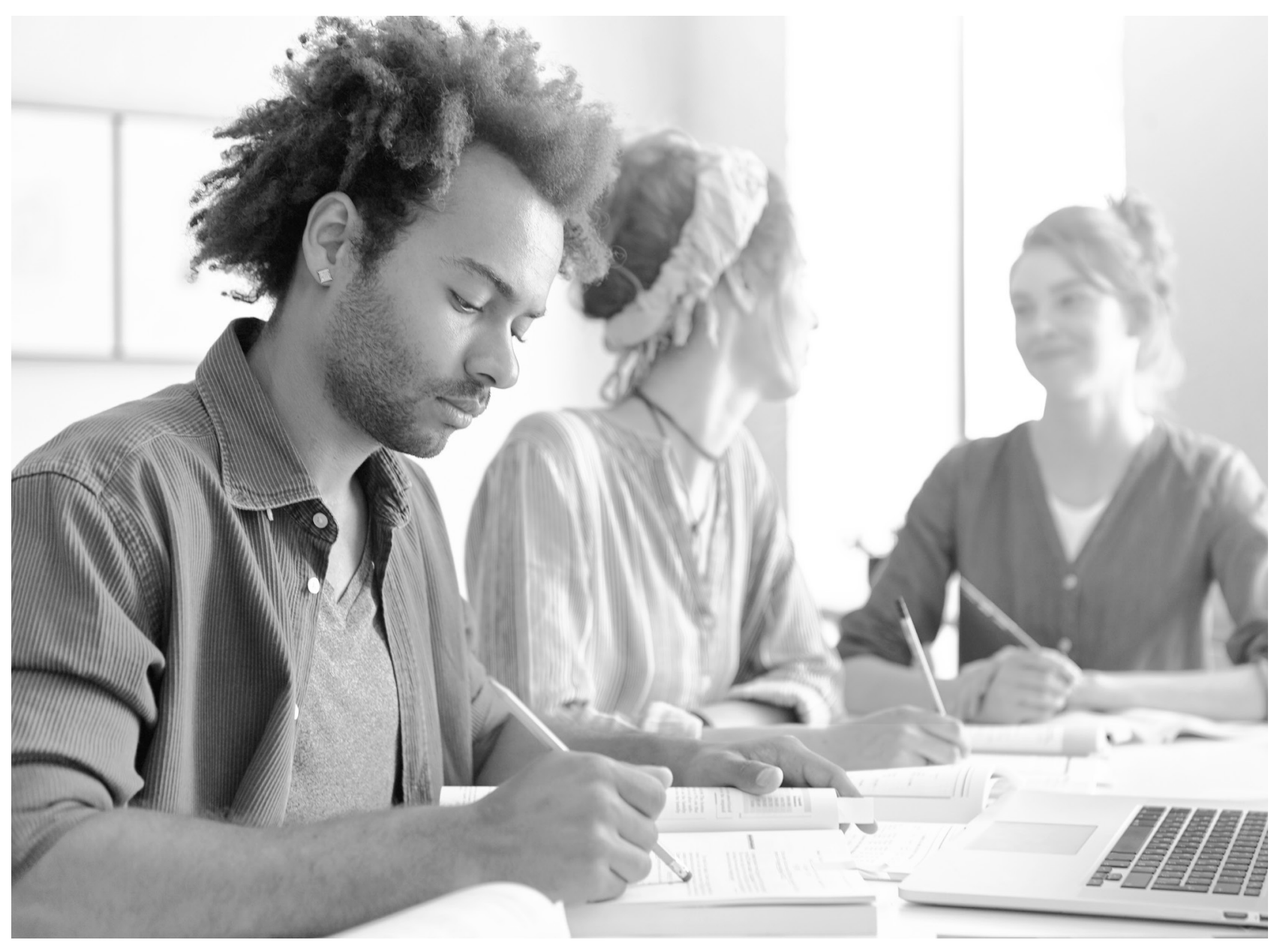




\title{
Estrategias didácticas para el fortalecimiento de la competencia de comunicación escrita en estudiantes universitarios ${ }^{1}$ Teaching strategies for strengthening the written communication competence in university students
}

\author{
María Nuria Rodríguez ${ }^{2}$, German Amaya Franky ${ }^{3}$, Socorro Astrid Portilla Castellanos ${ }^{4}$
}

Artículo recibido en marzo 30 de 2019; artículo aceptado en marzo 19 de 2020

Este artículo puede compartirse bajo la licencia Licencia Creative Commons Atribución-NoComercial-Compartirlgual 4.0 Internacional y se referencia usando el siguiente formato: Rodríguez, M., Amaya, G. y Portilla, A. (2020). Estrategias didácticas para el fortalecimiento de la competencia de comunicación escrita en estudiantes universitarios. I+D Revista de Investigaciones, 15 (2), 7-17. DOl: https://doi.org/10.33304/revinv.v15n2-2020001

\begin{abstract}
Resumen
Las evaluaciones externas se han convertido en uno de los retos de los sistemas educativos en el mundo. Colombia no es la excepción, razón que ha llevado a investigadores desde diferentes áreas del conocimiento a indagar y gestionar estrategias pedagógicas innovadoras que fortalezcan procesos de aprendizaje, especialmente en lo relacionado con la lectura y escritura. La presente investigación se fundamentó en el análisis estadístico de los resultados de las pruebas Saber 2014-2017 presentadas por estudiantes de una institución de educación superior de Bucaramanga, insumo relevante para la generación de estrategias que posibiliten el fortalecimiento del proceso lectoescritor a partir de la investigación-acción en el aula, teniendo en cuenta los procesos de diseño, implementación, evaluación, reflexión y rediseño en estudiantes desde el primer semestre académico. Los resultados de la investigación apuntan a relacionar las deficiencias en el proceso lectoescritor desde la educación inicial y las dificultades en la gestión de sus emociones con el rendimiento académico en la educación superior.
\end{abstract}

Palabras clave: Afectividad, enseñanza superior, lectura, lengua escrita, práctica pedagógica.

\footnotetext{
'Artículo de investigación, de enfoque cualitativo, de tipo de investigación-acción, resultado del proyecto de investigación culminado"Estrategias didácticas para el fortalecimiento de la competencia comunicación escrita en estudiantes de la Universidad de Investigación y Desarrollo (UDI)". perteneciente al área de ciencias sociales y humanas, subárea de educación, desarrollado en el grupo de investigación FIEDS, financiado por la Universidad de Investigación y Desarrollo (UDI), de la ciudad de Bucaramanga, (Colombia). Dirección: calle 9 n. ${ }^{\circ}$ 23-55, PBX: 6352525. Fecha de inicio: agosto de 2019. Fecha de terminación: diciembre de 2019.

${ }^{2}$ Doctorado en Pedagogía, Universidad del País Vasco. Adscrita al Grupo de investigación Educación y Lenguaje, Universidad Autónoma de Bucaramanga, de la ciudad de Bucaramanga (Colombia). Dirección: avenida 42 n. ${ }^{4} 48-11$. PBX 64361111. ORCID ID: https://orcid.org/0000-00026398-695X. Correo electrónico institucional: mrodriguez4@unab.edu.co.

${ }^{3}$ Doctorado en Procesos de Formación en Espacios Virtuales, Universidad De Salamanca. Adscrito al Grupo de investigación Pedagógica, Universidad de Pamplona, de la ciudad de Pamplona (Colombia). Dirección: km 1 vía Bucaramanga. PBX 5685303. ORCID ID: https://orcid.org/0000-0001-70509484. Correo electrónico institucional: german.amaya@unipamplona.edu.co.

${ }^{4}$ Doctorado en educación, Universidad de la Salle. Adscrita al Grupo de investigación FIELDS, Universidad de Investigación y Desarrollo (UDI), de la ciudad de Bucaramanga (Colombia): Dirección: Calle 9 n. 23-55. PBX: 6352525. ORCID ID: https://orcid.org/0000-0002-7580-5634. Correo electrónico institucional: sportilla2@udi.edu.co.
} 


\begin{abstract}
External evaluations have become one of the worldwide challenges of educational systems, and Colombia is no exception. This is the reason that has led researchers from different areas of knowledge to investigate and manage innovative pedagogical strategies that strengthen learning processes, especially in relation to reading and writing. This research was based on the statistical analysis of the results of the Tests Saber Pro 2014-2017, presented by students from a higher education institution in Bucaramanga, a relevant input for the generation of strategies that enable the strengthening of the reading-writing process from the action research in the classroom, taking into account the processes of design, implementation, evaluation, reflection and redesign in students from the first academic semester. The results of the research aim to relate the deficiencies in the reading-writing process from initial education and the difficulties in managing their emotions with academic performance in higher education.
\end{abstract}

Keywords: Affectivity, higher education, reading, written language, pedagogical practice.

\section{Introducción}

Fortalecer competencias comunicativas escritas y orales se ha convertido en un reto en todos los niveles del sistema educativo, debido a la pertinencia de estas en la apropiación de aprendizajes significativos en el relacionamiento social y en las opciones que ofrecen para manifestar sentimientos y emociones. Lamentablemente los estudiantes no asumen la importancia de adquirir dichas competencias; consideran que leer y escribir algunos símbolos lingüísticos es suficiente para evidenciar el dominio de estas habilidades.

En los participantes de la investigación, estudiantes de primer semestre, se detectaron dificultades en el proceso lectoescritor, aspecto que, si bien estos reconocen, evidencian poco interés en resolver, debido a que consideran el desarrollo de dichas habilidades como un problema de la básica primaria y secundaria, con lo que dejan de lado aspectos como letra ilegible y dificultades gramaticales, semánticas, sintácticas y para expresarse en público. Así mismo, hay una deficiente comprensión de lectura y desconocimiento de signos de puntuación, tanto al leer como al escribir. Este escenario es visible al presentar las pruebas externas Saber Pro al culminar la universidad.

En el departamento de Santander los resultados no difieren del resto del país. Durante el proceso investigativo se realizó la comparación de resultados alcanzados por estudiantes de una institución de educación superior de la ciudad de Bucaramanga, Colombia, en las pruebas genéricas de competencia comunicativa y lectura crítica de Saber Pro, durante los periodos 2014-2017, con los de estudiantes en el mismo grupo de referencia de otras universidades de Bucaramanga. De esto se logró establecer que no existe gran diferencia en los resultados, pues en su mayoría se encuentran por debajo de la media nacional, tanto en competencia escrita como en lectura crítica.
El presente documento es el resultado del proceso de investigación aplicada, a partir del proyecto sobre estrategias didácticas para el fortalecimiento de la competencia de comunicación escrita en estudiantes de educación superior. El estudio se enfoca en el análisis de las competencias comunicativas escritas y en la búsqueda de estrategias didácticas que brinden una solución temprana a las deficiencias que presentan los estudiantes desde el primer semestre académico, con el propósito de mejorar no solo en la presentación de las pruebas externas, sino como mecanismo para la apropiación de aprendizajes significativos en todas las áreas del conocimiento.

Con fundamento en lo mencionado, se formuló la siguiente pregunta: ¿Cómo fortalecer las competencias comunicativas escritas en estudiantes de educación superior de Bucaramanga? Este interrogante se despejó a partir del análisis de los resultados alcanzados en las pruebas externas, durante el periodo 2014-2017, en competencias comunicativas escritas y lectura crítica, en el análisis de pruebas diagnósticas, entrevistas y observación directa.

Así mismo, en lo comportamental se detectaron jóvenes desmotivados, distraídos, agresivos, apáticos y sin un propósito de vida. Este factor se constituyó en la principal categoría emergente del proceso investigativo, puesto que se logró evidenciar la relación de las emociones con la falta de interés académico, la pasión por aprender y los resultados evaluativos, tanto en cursos del programa de formación como en las pruebas Saber Pro; es decir, la relación directa entre emociones y aprendizaje.

El objetivo de la investigación se orienta al diseño de estrategias didácticas para fortalecer competencias comunicativas. A partir del enfoque metodológico de investigación-acción, se reflexionó, desde una mirada crítica, sobre la práctica pedagógica del maestro y la actitud de los estudiantes frente a las estrategias didácticas propuestas. Las estrategias se implementaron durante el 
periodo académico en el que estuvo activa la ejecución del proyecto, y fueron objeto de continua evaluación, reflexión y reconfiguración. Se logró comprobar que las actividades lúdicas que impliquen movimiento y trabajo en equipo estimulan la apropiación de aprendizajes significativos (Cassany, 2006a, 2006b). Para este estudio se considera importante la investigación educativa, en la medida que permite establecer argumentos que validan el proceso de construcción del concepto de enseñanzaaprendizaje desde una perspectiva crítico-social de la investigación. Desde este punto de vista, el concepto se asume como la posibilidad de reconfigurar los contextos a partir de las experiencias y la práctica.

\section{Fundamento teórico}

El fundamento teórico de la investigación estuvo sustentado en referentes como Cassany $(2006 a, 2006 b)$ y Solé $(2010,2012)$, con relación al proceso didáctico para la adquisición de competencias comunicativas orales y escritas. Además, se abordó la noción de competencias comunicativas y los indicadores de desempeño esperados para el nivel profesional en las habilidades comunicativas escritas, así como una exploración documental a través de la web, con el propósito de establecer qué proyectos, programas o estrategias relacionadas con la competencia comunicativa y las pruebas Saber Pro se han implementado en los ámbitos internacional, nacional y local. Inicialmente, se plantea la investigación realizada por Alvarado (2012), que se desarrolló con estudiantes del curso Lengua Española, a través de talleres didácticos y bajo la metodología de investigación-acción. El propósito de este estudio fue mejorar las dificultades escritoras de los estudiantes, en cuanto a la gramática, redacción y composición de texto, bajo los postulados de macrorreglas propuestas por Cassany (2006a, 2006b). Esta investigación guarda relación con el presente estudio, dado que se realiza con 72 estudiantes del curso Técnicas de Comunicación Oral y Escrita, bajo el diseño metodológico de la investigaciónacción, con el enfoque de macrorreglas para la escritura (Cassany, 2006a), y la propuesta pedagógica se desarrolla a partir de talleres lúdicos.

Otra investigación consultada fue la de Rincón (2014), quien presenta un artículo de investigación denominado "Formación en competencias comunicativas en educación media y su incidencia en la educación superior", avance de investigación del trabajo de grado de Especialización en Pedagogía de la Educación Superior. En el documento se analiza el estado de las competencias comunicativas en Colombia, a partir de los resultados de las pruebas Saber 2012-2013 en estudiantes de educación media y su incidencia en el ingreso a la universidad. La investigación arrojó como resultado el bajo nivel de los estudiantes en competencias comunicativas, aspecto que se evidencia en los diferentes procesos académicos en la universidad objeto de estudio. Por otra parte, la autora afirma que las dificultades comunicativas no solo influyen en la adquisición del conocimiento, sino en las habilidades para relacionarse con compañeros, maestros y familiares. Una de las conclusiones más importantes del artículo revisado corresponde al planteamiento de articular la educación media y superior para detectar de manera temprana y oportuna las falencias de los estudiantes, principalmente en proceso lectoescritor; además, considera pertinente trabajar de manera sincronizada para lograr mitigar las falencias con las que ingresan los estudiantes a la educación superior $y$, por supuesto, los bajos resultados en las pruebas Saber al culminar su carrera profesional (Simancas y Utria, 2013).

Por su parte, Sanchez y Brito (2015) presentan la propuesta para la creación de un Centro Permanente de Lectura Comprensiva, aula y taller creativo, para desarrollar habilidades comunicativas en los estudiantes de primer semestre que ingresen a la Universidad de la Costa. Proponen la necesidad de realizar una prueba diagnóstica en habilidades comunicativas lectoras y escritoras que logre determinar las necesidades de fortalecimiento de procesos y clasifique por niveles a los estudiantes, quienes deben asistir durante su proceso de formación profesional al aula taller. Los autores mencionan que, en una prueba piloto realizada a la población de primer ingreso a la universidad, se pudo establecer que solo el $32 \%$ de los estudiantes se encontraba en un nivel medio de competencias comunicativas; más del $90 \%$ de los estudiantes manifiesta no haber leído un libro completo, y más del $45 \%$ de los estudiantes reconoce tener dificultades al momento de escribir relacionadas con la legibilidad de la letra y la gramática. El estudio citado guarda relación con la intención investigativa del proyecto presentado en este artículo, ya que se considera de vital importancia generar espacios desde el primer semestre para fortalecer las competencias comunicativas y dar solución a tiempo a las falencias presentadas por los estudiantes al ingresar a la educación superior. Esta situación beneficia los aprendizajes significativos y mejora los resultados al presentar las pruebas externas.

Así mismo, Castaño y Echenique (2017) presentan en su artículo los resultados del proyecto de investigación "Estrategias didácticas para el desarrollo transversal de la competencia comunicativa en la formación de estudiantes de educación superior". La investigación surge por la necesidad de presentar una propuesta institucional que responda a las necesidades de formación transversal de competencias en todos los programas profesionales que ofrece la universidad. Presentan una propuesta de intervención en cuatro fases. La investigación planteada puede ser replicada en universidades de Santander, con el desarrollo de pruebas de talentos para cada programa, concursos de cuento y oratoria, entre otros. 
La investigación de Benítez y Rincón (2016) tuvo como propósito analizar cómo se enseñan competencias comunicativas orales y escritas en estudiantes de pregrado del programa Contaduría Pública, en la Universidad Pontificia Bolivariana, como parte del proceso de formación integral esperada para un profesional del área de la contaduría. El estudio se llevó a cabo bajo un enfoque cualitativo con docentes de planta y de cátedra que ofrecen servicio al programa. Con los participantes se indagó acerca de las diferentes estrategias utilizadas desde todos los cursos para fortalecer el proceso lectoescritor de los estudiantes, independientemente del curso institucional de técnicas de comunicación. Se evidenciaron falencias relacionadas con didáctica en el aula, desconocimiento en la elaboración de algunos tipos de texto, espacios curriculares para fortalecer el proceso, inclusión de estrategias como presentación de informes sin la utilización del computador, lectura de libros y artículos de investigación, presentaciones orales, debates, entre otras. Esta investigación se tomó como referente, dada la necesidad de proponer, para universidades de Bucaramanga, Santander, una propuesta de fortalecimiento docente en el proceso lectoescritor, así como de generar estrategias didácticas que les permitan a todos los docentes, desde sus áreas de conocimiento, fortalecer dicho proceso en los estudiantes.

Por otra parte, surgió como categoría emergente la influencia de los estados emocionales en el rendimiento académico de los estudiantes, ya que se pudo establecer que la cercanía del maestro, la constante motivación, el aumento de la autoestima y la empatía generan efectos positivos sobre la adquisición de aprendizajes significativos por parte de los estudiantes (Goleman, 2010). En este tema se consultaron estudios investigativos como el de Fragoso-Luzuriaga (2015), quien realizó un análisis de los términos inteligencia emocional y competencias emocionales, desde sus orígenes, y logró establecer las diferencias y similitudes; destaca también la importancia de desarrollar este aspecto en estudiantes de educación superior, profesionales que, culminado su proceso de formación, hacen parte de la fuerza laboral y económica de los países. Esta investigación contribuyó con el presente estudio al presentar el desarrollo histórico de los dos términos y diversos autores que fueron tomados como referentes.

Por su parte, el artículo de García Retana (2012) presenta la importancia de promover y manejar adecuadamente estados emocionales en el aula y la influencia del maestro en dicho proceso. El autor considera de vital importancia para el proceso de enseñanza-aprendizaje, además de las habilidades cognitivas, promover el desarrollo emocional como parte de la motivación hacia el aprendizaje, autoestima, resolución de problemas y relaciones interpersonales. Considera también relevante un clima de aula agradable para que se produzcan aprendizajes significativos. Esta investigación aportó valiosa información al presente estudio, ya que propició incluir técnicas de manejo emocional en el aula, haciendo evidente el cambio de actitud por parte de los estudiantes.

Por otra parte, Bisquera Alzina (2003) considera necesario promover el desarrollo de habilidades de vida y bienestar bajo el diseño curricular de programas que tengan en cuenta formar desde la integralidad, teniendo en cuenta las emociones y sentimientos de los estudiantes. Esta teoría se considera soporte para el presente estudio, ya que los componentes propuestos por el autor se observaron en el grupo de estudiantes vinculados al proyecto.

\section{Metodología}

La investigación se desarrolló bajo un enfoque cualitativo y de tipo de investigación-acción en el aula.

Este tipo de estudio posibilita la selección del escenario en el que se registran sistemáticamente las experiencias de aprendizaje en el aula de clase y se identifican las personas que colaboran con el suministro de información sobre su experiencia en el trabajo académico.

La investigación-acción, concebida como una propuesta metodológica que destaca un proceso cíclico constituido por fases a partir de la observación, diseño, implementación, evaluación, reflexión y rediseño, se consideró apropiada, ya que, además de posibilitar opciones de mejora a la problemática detectada en los estudiantes, contribuye al perfeccionamiento de la práctica pedagógica del maestro y las estrategias didácticas que le permiten promover aprendizajes significativos en los estudiantes.

Para el desarrollo de la investigación se asumieron las siguientes fases:

- Planteamiento de la idea inicial

- Identificación de la problemática

- Diseño de un plan de acción

- Implementación del plan

- Valoración de resultados

- Rediseño

- Evaluación y reflexión

- Presentación de producto

La población participante estuvo representada en 466 estudiantes del curso Técnicas de Comunicación Oral y Escrita matriculados en el Departamento de Humanidades para el segundo semestre de 2018, de los cuales 131 estudiantes participaron de manera voluntaria en las entrevistas y grupos focales. Por su parte, la prueba 
diagnóstica fue aplicada a 72 de estos estudiantes.

Para la recolección de la información se utilizaron los siguientes instrumentos: un taller diagnóstico basado en el relato, un ejercicio para establecer las habilidades gramaticales, una entrevista, una encuesta para complementar la información cualitativa recogida y la observación directa de actitudes; además, una encuesta para determinar habilidades relacionadas con las competencias emocionales.

Las categorías de investigación tenidas en cuenta para la estructuración del proceso investigativo fueron estrategias pedagógicas, experiencias significativas, dificultades en el proceso lectoescritor, emocionalidad y niveles gramaticales.

\section{Proceso de recolección y análisis de la información}

El proceso de análisis de la investigación cualitativa es trasversal. Las entrevistas a los estudiantes del curso Técnicas de Comunicación Oral y Escrita de una universidad de Bucaramanga se registran en forma magnetofónica, para contar con una memoria de las conversaciones que permita recuperar la construcción de sentidos que hace el investigador de la experiencia. Los registros sonoros se transcriben a fin de facilitar la manipulación de los datos por parte del investigador. Luego de la transcripción de los datos, el investigador elabora un cuadro en el que se ubica el contenido de cada una de las entrevistas y otros materiales. En ese cuadro se asigna una casilla para el microanálisis del relato, que consiste en que el investigador identifique los vacíos de la información de las entrevistas grabadas y formule las preguntas que permitan precisar o profundizar esos datos. Este ejercicio investigativo se llevó a cabo con la colaboración de los estudiantes, como parte del taller de la entrevista utilizado como estrategia didáctica de aprendizaje y como herramienta de recolección de la información.

Realizadas estas entrevistas complementarias, el investigador inicia el proceso de codificación abierta, mediante la lectura cuidadosa de cada uno de los fragmentos de las entrevistas y la asignación de una etiqueta o código de acuerdo con el sentido de las frases. Con base en las etiquetas de la codificación descriptiva, el investigador empieza a reordenar la información de acuerdo con las construcciones de sentido que surjan de la reunión de etiquetas que tienen el mismo nombre.

Esa reordenación de las etiquetas sirve para la elaboración de los memorandos, en los que se registran las propiedades y dimensiones particulares de un concepto, que surge de la misma significación que se les atribuye a los colaboradores. Es así como la interpretación está guiada por los memorandos conceptuales, utilizando las propiedades identificadas, que permitieron establecer los significados dominantes que sirvieron para la construcción de los hallazgos.

\section{Hallazgos de la investigación}

Se tomaron como referencia para el estudio inicial de la propuesta de investigación los resultados alcanzados por los estudiantes en la prueba de comunicación escrita y lectura crítica durante los últimos tres años; es decir, el periodo comprendido entre 2014-2017. La información se analiza cuantitativa y cualitativamente, a fin de establecer qué aspectos se deben fortalecer en la población estudiantil con fundamento en los resultados tomados del Icfes (2017a), agregados en 2017, de las pruebas Saber.

Se consideraron los periodos por separado, debido al ajuste que se presentó en 2016, con relación a la presentación de resultados. Así mismo, se muestra la tendencia en cada una de las competencias, tomando como referencia el último año de observación. A partir de 2015, los parámetros de evaluación cambiaron: hasta 2014 la ubicación de los estudiantes estaba organizada por quintiles; a partir de 2016, por niveles.

Teniendo en cuenta la valoración propuesta por el Icfes para categorizar los estudiantes, se pudo establecer que durante el periodo 2014-2015 los estudiantes estuvieron en su mayoría ubicados en el quintil bajo y regular. Unos pocos programas lograron estar en bueno y muy bueno; es decir, la mayoría de los programas académicos estuvieron entre 9 y 10,7 puntos, quintil 2,3 y 4. Para 2016 y 2017 , tanto en lectura crítica como en comunicación escrita, los resultados alcanzados por los estudiantes los ubicaron en los niveles 2 y 3 .

En la competencia lectura crítica, es muy importante tener en cuenta los elementos que se evalúan en el módulo propuesto por el Icfes (2017b). Competencias por considerar:

1. Identificar y entender los contenidos locales que conforman un texto: Esta competencia se refiere a la capacidad de comprender palabras o expresiones en un texto y relacionarlo con el contexto en que vive o la realidad local, nacional o global. Así mismo, logra identificar ideas principales y secundarias, personajes, tipo de texto, entre otros.

2. Comprender cómo se articulan las partes de un texto para darle un sentido global: En este aspecto es relevante comprender la relación semántica de los elementos que componen un texto, diferentes tipos de voces, reconoce textos continuos y discontinuos.

3. Reflexionar a partir de un texto y evaluar su contenido. 
Al realizar un análisis comparativo entre los resultados obtenidos por los estudiantes, se pudo evidenciar que el programa de Publicidad se ha destacado tanto en lectura crítica como en comunicación escrita en diversos periodos considerados en el presente estudio. Desde la dirección del programa académico, se logró establecer que se realizan acciones como plan de lectura, ejercicios de comprensión lectora, consultas y exposiciones, entre otras acciones que se suponen interfieren de manera positiva en los resultados alcanzados por los estudiantes.

Al comparar los resultados alcanzados por los estudiantes de Publicidad de la institución analizada con otras instituciones en el mismo grupo de referencia, se logró establecer que la universidad objeto de este estudio se encuentra ubicada en el mismo nivel de las otras universidades: a escasos 3 puntos de la universidad con más alto promedio para este grupo de referencia en 2017. Situación que, aunque no la deja en desventaja en el ámbito local, si requiere una solución inmediata, ya que la ubicación no sobrepasa el nivel 2 en el ámbito nacional.

En 2017 se evidencia una distancia considerable en las dos habilidades de comunicación escrita y lectura crítica, con los resultados alcanzados por estudiantes de otras universidades de Santander en el mismo grupo de referencia. Al analizar estos datos con los estudiantes que alcanzaron los resultados más bajos en el mismo periodo, la distancia se hace aún más amplia.

La mayoría de los programas por grupo de referencia en comunicación escrita en 2017 estuvo por debajo de los 150 puntos, sin alcanzar la media nacional.

\section{Caracterización de participantes}

Tomando como referentes los aspectos propuestos por el Icfes (2016) para la evaluación del módulo de comunicación escrita y el análisis de los resultados alcanzados por los estudiantes en los 11 programas evaluados durante el periodo 2014-2017, se realizó la caracterización del grupo de 72 participantes pertenecientes al curso Técnicas de Comunicación Oral y Escrita.

Inicialmente se aplicaron talleres diagnósticos para determinar el nivel de competencia comunicativa en que se ubicaban, prestando también atención a aspectos gramaticales y a la legibilidad de la letra (ver Tabla 1).

Los resultados del primer taller mostraron la necesidad de fortalecer aspectos gramaticales, sintácticos y semánticos. De los 72 estudiantes, 54 no tienen claridad sobre la definición de diferentes tipos de palabras: sustantivo, adjetivo, verbo, adverbio, conjunciones, preposiciones, y difícilmente realizan un análisis morfológico. Así mismo, se evidencia dificultad en los niveles fonológico, semántico y sintáctico.
En cuanto a los mecanismos de coherencia y cohesión evaluados en las pruebas Saber, se pidió el escrito de un relato narrativo-descriptivo relacionado con la historia del primer cuaderno. La mayoría de los estudiantes escribió entre 4 y 8 renglones. Solo 6 de los 72 estudiantes escribieron un texto de más de 8 párrafos. En este ejercicio se identificaron dificultades relacionadas con letra ilegible, deficiente ortografía, acento ortográfico, ambigüedades, redundancias y poca organización de las ideas.

Tabla 1

Aspectos evaluados en los talleres diagnósticos

\begin{tabular}{ll}
\hline Producción & Nivel sintáctico: Organización del texto \\
escrita & (jerarquización de las ideas) \\
& Nivel semántico: Propiedades de cohesión, \\
& coherencia y adecuación \\
& Nivel pragmático: Lenguaje e intención \\
& comunicativa \\
& Nivel gramatical: Legibilidad, ortografía \\
\hline
\end{tabular}

Fuente: Autores.

\section{Análisis de las entrevistas a estudiantes}

Las entrevistas a estudiantes se realizaron teniendo en cuenta las categorías de investigación propuestas en la Tabla 1. Tomando como referencia las categorías de investigación se analizaron las entrevistas aplicadas a 72 estudiantes de primer semestre del curso Técnicas de Comunicación Oral y Escrita, y 57 encuestas a estudiantes del mismo curso en la jornada diurna. Se logró establecer lo siguiente:

\section{Estrategias pedagógicas}

Los estudiantes afirman que fueron muy pocas las experiencias significativas lúdicas que recuerdan de sus inicios en el proceso lectoescritor. La mayoría, tanto en las entrevistas como en las encuestas, sostiene que las planas fueron la estrategia más utilizada por los maestros y padres de familia.

Por otra parte, trascribir artículos del periódico o escritos de otros libros, dictados y llenar fichas de libros de español fueron las principales estrategias utilizadas por los maestros para enseñar a escribir. Algunos estudiantes afirman que creían que ese trabajo no tenía sentido, ya que, en la mayoría de los casos, los profesores no los revisaban ni tampoco explicaban cómo hacerlos.

Se logró establecer que los castigos, golpes y amenazas hicieron parte de la estrategia lectoescritora en la primera infancia. En las entrevistas se encontraron comentarios como:

Siempre he tenido mala letra y durante mi infancia 
me la pasé castigado las horas de recreo tratando de mejorar la letra, pero aun así no pude, confundía las vocales y las letras $b$ con $d$, y $p$ con $q$, aun lo hago. La profesora me puso a transcribir un libro de las noches oscuras y me dio un mes para hacerlo, pero como molestaba en clase, adicionalmente, me hizo firmar observación y me castigo una semana escribiendo en un cuaderno doble línea -debo respetar a mi compañera. Siempre que me ponían hacer planas, empezaba bien, pero me cansaba y hacia las planas de mala gana, mi profesora me castigaba en la escuela y mi mama en la casa me daba correa. La profesora hacia comentarios desagradables sobre mis calificaciones $\mathrm{o}$ trabajos en frente de mis compañeros, en vez de motivarme a aprender un poco más, lo que hizo fue desmotivarme en el colegio. El profesor promovía que mis compañeros se burlaran de mi porque no podía hablar en público, me pasaba a propósito 5 .

Estos comentarios y otros permiten reflexionar acerca de las acciones que se tomaron para iniciar el proceso lectoescritor como una experiencia traumática y desmotivadora que, lejos de acercar al aprendizaje, generó situaciones de ansiedad y temor por las consecuencias de equivocarse. Los castigos y las planas no lograron dar solución a las problemáticas que algunos presentaban en su infancia, pues ellos reconocen que en esta nueva etapa de escolaridad conservan dichas problemáticas. Trascribir un texto sin un sentido para el estudiante, además de alejarse del objetivo pedagógico supuesto de mejorar la letra, generó apatía hacia el proceso escritor, más aún cuando la profesora revisa la cantidad de cuadernos trascritos y no su contenido. Muchos de los estudiantes refieren que durante la infancia presentaron problemas de aprendizaje, pero que jamás recibieron atención profesional. Los padres consideraban que no escribían, o lo hacían mal por rebeldía; y algunos maestros los golpearon o ridiculizaron, lo que los afectó psicológicamente.

El texto guía más recordado fue Nacho lee. En uno de los textos se cita los siguiente:

Una de las experiencias que marco mi vida en el colegio especialmente en la parte lecto-escritura fue el libro de Nacho, los profesores nos ponían a leer, a interpretar, todo lo que tenía que ver pues con las imágenes y todo lo que traía el libro y ahí fueron mis bases para aprender a leer y a escribir. Mi mamá me compró el libro de Nacho lee, lo que más recuerdo son las imágenes y las letras grandes

\footnotetext{
${ }^{5}$ Información tomada de manera literal de los informes de entrevista presentados por los estudiantes, o de su ampliación textual en el análisis de la información. Los testimonios de estudiantes que se citen en adelante se ajustarán a este mismo criterio de escritura.
}

en las páginas. Me ponían hacer las planas del libro Nacho lee y para mí era una tortura.

Estas apreciaciones llevan a reflexionar acerca de la efectividad de utilizar un texto guía en la primera infancia para iniciar el aprendizaje del proceso lectoescritor. Los efectos se están viendo actualmente frente a estudiantes desmotivados. Sería importante recapitular acerca de los intereses de los niños y presentar lecturas que generen el deseo de leer y profundizar de manera autónoma en los diferentes temas de interés.

Por otra parte, se considera pertinente atender oportunamente las diferencias de aprendizaje, dificultades cognitivas y físicas de los estudiantes, ya que la falta de acompañamiento durante los primeros años de aprendizaje repercute de manera negativa en toda la escolaridad, como se ha podido evidenciar en el grupo de participantes del proyecto.

En el diagnóstico la mayor dificultad detectada estuvo relacionada con letra ilegible, signos de puntuación, lateralidad en el texto y dificultad para diferenciar entre sustantivo, adjetivo y verbo. Por otra parte, dos de los estudiantes presentan dificultades de aprendizaje, atención dispersa, y en ocasiones intercambio de letras, principalmente $b$ y $d$, y $p$ y $q$.

Por otra parte, se pudo observar mezcla entre mayúsculas y minúsculas, espacios desiguales entre letras; algunos estudiantes separan palabras en silabas, y un alto porcentaje de los estudiantes presenta dificultad en el agarre del lapicero, entre otras dificultades.

\section{Manejo emocional frente al proceso de enseñanza- aprendizaje de la escritura}

Durante el desarrollo del proyecto de investigaciónacción, se realizó la experiencia dialógica con los estudiantes, para establecer su estado emocional frente a los bajos resultados presentados en las evaluaciones.

Algunos de los estudiantes afirmaron que durante toda su escolaridad habían presentado dificultades en la escritura; las tortuosas tareas de trascribir textos y realizar planas sin sentido no lograron motivar al estudiante. Consideran en algunos casos innecesario aprender a escribir correctamente, ya que el apoyo de las tecnologías, tanto en la gramática como en la legibilidad del texto, les permite presentar informes. Afirman que se sienten más cómodos enviando la información vía web que entregando de su puño y letra en físico. Frente a esta situación, fue necesario mostrar las ventajas de adquirir habilidades escritoras, pues el equipo de cómputo les permite presentar el informe, pero la redacción y el sentido del texto son tarea propia del estudiante. 
Por otra parte, se evidenció que algunos estudiantes guardaban resentimiento hacia los maestros y padres de familia, de quienes recibieron golpes y los pusieron en ridículo frente a los compañeros cuando cometían errores o se les dificultaba escribir o transcribir lo impuesto por el maestro. Abiertamente mencionaron que cuando van a escribir se bloquean, sienten inseguridad, nervios, no logran tomar apuntes de lo expuesto por el profesor, ya que no cuentan con la agilidad requerida para captar las ideas rápidamente y transcribirlas de acuerdo con su comprensión.

Frente a algunos retos de producción textual, se notó apatía por algunos estudiantes al devolverles los escritos y pedirles que intentaran organizar las ideas nuevamente. Manifestaban que no se sentían capaces. Inicialmente algunos preferían recibir una baja calificación que corregir los textos, pero esta situación fue variando a medida que el docente motivó a los estudiantes, alentándolos con palabras que estimularon su estima, como "inténtalo de nuevo"; "eres inteligente"; "confió en que puedes hacerlo"; "escribe algo pequeño, lo mejor que puedas, y te lo califico"; "cuéntame que es lo que te pasa"; "escribe sobre las cosas que te gustan, que te hacen feliz o triste, sobre cuáles son tus metas", entre otras.

La información recogida de los 72 estudiantes con relación a las habilidades emocionales permitió identificar algunas dificultades que requieren atención:

En la autoconciencia emocional, la mayoría de los estudiantes menciona que casi siempre logra identificar sus emociones, pero que difícilmente puede evitar estallar en cólera o en euforia. Para 14 estudiantes este aspecto se considera indiferente; para ellos no es relevante tener conocimiento de los momentos que anteceden a una emoción, y simplemente la experimentan sin importar el sitio o las personas con las que se encuentren. Afirman que frente a situaciones de enojo difícilmente recuperan la calma; en ocasiones este sentimiento ha llegado a terminar con amistades o a generar conflictos familiares cuando se presentan altercados con miembros de la familia (Goleman, 2010).

Un aspecto positivo por reconocer en el grupo de estudiantes, frente a las explicaciones sobre la importancia de identificar, manejar las emociones y hacerse consientes de estas para tener una mejor calidad de vida, fue el entender que asimilar la información y reconocer cómo el no hacerlo afectaban las relaciones interpersonales en el aula y fuera de ella, además de los resultados académicos.

En cuanto a la empatía y competencia social, nueve de los estudiantes consideraron que no contaban con esta habilidad. No están interesados en generar relaciones interpersonales en el aula, de forma que prestan poca atención a la imagen que tienen sus compañeros de ellos; consideran que es mejor el trabajo individual y evitar en lo posible vincularse más allá del aula con los compañeros de curso. Seis de los entrevistados afirman que desde siempre se han sentido inseguros, tímidos, incapaces de entablar conversación, hablar en público o participar en la clase; afirman que el miedo a la burla o reprensión por parte del docente les impide preguntar o participar en las actividades académicas; se sienten ignorados, invisibles para el profesor y sus compañeros. Tres de los estudiantes mencionaron que no sienten la confianza suficiente con los miembros de la familia o algún amigo para expresar sus sentimientos, preocupaciones y miedos.

Por otra parte, se logró evidenciar que la mayoría de los estudiantes afirma contar con buenas relaciones interpersonales, tener habilidad para comunicarse con sus compañeros y familiares, así como de trabajar en equipo; aunque se evidenció que ya existen grupos conformados. Sin embargo, pese a las múltiples estrategias en busca de distribución de nuevos equipos, o en busca de que fluya el trabajo con cualquier compañero, fue difícil lograr esta diversidad. En cuanto al grado de tolerancia emocional, ninguno se siente en capacidad de soportar estados emocionales de otras personas. La mayoría de ellos afirma que no controla la calma, la paciencia y la sensibilidad para ayudar a otro compañero o maestro en estados emocionales alterados.

La mayoría considera que lograr buenas relaciones interpersonales y trabajo en equipo colaborativo influye de manera positiva en el aprendizaje; pero un aspecto en el que se recalcó permanentemente fue en la actitud del maestro: afirman que es de vital importancia la empatía que alcance con el grupo para que se genere confianza y motivación por la asignatura trabajada.

En el estudio se logró determinar la dificultad de escucha que tienen los estudiantes. En ocasiones es necesario llamar la atención de manera permanente durante las explicaciones del profesor o en las intervenciones de los compañeros de curso.

Lo anteriormente mencionado evidencia el escaso autocontrol por parte de algunos estudiantes. Además de ser observado por el maestro, los estudiantes lo confirman. Estos mencionan que se sienten cansados con regularidad, trabajan durante todo el día y, al llegar al aula de clase, se desconcentran con facilidad, en ocasiones pensando en algunas tareas laborales pendientes, en situaciones emocionales experimentadas durante el día, o en actividades académicas por realizar para la clase siguiente.

La mayoría de los estudiantes afirmó que, aunque 
sienten motivación por aprender y culminar exitosamente la carrera profesional, algunas situaciones emocionales los afectan, lo que les impide alcanzar sus expectativas académicas. En tres de los estudiantes se observó constantemente pensamientos negativos, desmotivadores y relacionados con la baja autoestima; consideran que todo lo que hacen no sirve para nada, se refugian en el bajo nivel socioeconómico para justificar el bajo rendimiento académico obtenido.

En relación con la autoconfianza, la mayoría de los estudiantes manifiesta contar con esta habilidad; son capaces de relacionarse con los demás fácilmente y expresar sus puntos de vista. En el grupo de estudiantes observado, cuatro de estos mencionan que nunca - casi nunca se sienten capaces de expresar sus inconformidades; no están preparados para tomar iniciativa en una actividad o entablar una conversación: requieren de las indicaciones de los padres, jefes o profesores para hacerlo.

En resumen, se pudo establecer la estrecha relación entre el estado emocional y la adquisición de aprendizajes significativos. Es de vital importancia reconocer las diferencias individuales, flexibilizar los currículos, aprender a manejar adecuadamente nuestras emociones como maestros y generar espacios de motivación a los estudiantes para que se sensibilicen acerca de la importancia de desarrollar procesos cognitivos y emocionales que les permitan la adquisición de aprendizajes significativos y el logro de procesos académicos exitosos.

En el grupo observado, se detectaron estudiantes con problemas de aprendizaje, baja autoestima, desmotivación, falta de relacionamiento, dificultad de escucha y de lectoescritura.

Las estrategias pedagógicas implementadas a partir de la investigación-acción lograron aproximar de manera asertiva a algunos estudiantes al aprendizaje significativo.

\section{Conclusiones}

En la interpretación y búsqueda de soluciones a las problemáticas presentadas en el proceso lectoescritor, se han llevado a cabo innumerables estudios, pero muy pocos relacionan el aprestamiento inicial en la primera infancia con las dificultades en la educación terciaria. La presente investigación, además de resaltar algunos aspectos didácticos y metodológicos en la práctica del maestro y las estrategias de enseñanza utilizadas en el aula, evidencia la importancia del manejo emocional y el reconocimiento de estas habilidades en la apropiación de lenguaje oral y escrito, aspecto fundamental para la adquisición de conocimientos en todas las áreas del conocimiento.
El estudio destaca la importancia de realizar un correcto aprestamiento del proceso lectoescritor durante la primera infancia, esto es, la utilización de estrategias pedagógicas lúdicas y recreativas que enamoren al estudiante de la lengua castellana; además de sensibilizar a los maestros acerca del manejo emocional, generar empatía, confianza y seguridad en los estudiantes. Si no se trabajan estos aspectos de manera adecuada durante la educación inicial y la básica, se reflejan de manera negativa en la universidad. En muchos casos son motivo de deserción, dado que el estudiante siente frustración frente a la dificultad de asimilar información teórica en la que se requieren habilidades lectoescritoras.

La propuesta de intervención pedagógica implementada con los 72 estudiantes objeto de estudio mostró mejores resultados a partir del acompañamiento del maestro, quien atendió habilidades emocionales y motivó permanentemente, lo que permitió que los estudiantes expresaran libremente sus puntos de vista, además de la utilización de lecturas que apuntaron al fortalecimiento de valores. Se utilizó el texto Los cuatro acuerdos, de Miguel Ruiz, para el análisis reflexivo acerca de algunos comportamientos asumidos por los jóvenes que afectan la sana convivencia en el aula, aspecto que guarda relación con el manejo emocional, categoría emergente en la presente investigación.

El análisis del texto permitió a los estudiantes reflexionar sobre las posturas que asumimos, algunas de estas de arraigo cultural ancestral. Se revisaron por binas las producciones escritas, evidenciando notoria mejoría en la redacción y ortografía, así como en la expresión oral.

Con este estudio se logra comprender que es indispensable, antes de enseñar contenidos teóricos, sensibilizar a los estudiantes acerca de su aplicación en la cotidianidad y el aporte de estos a la formación profesional; es decir, el aporte que brinda lo que se aprende para la vida.

Se reconoció la importancia de vincular en las actividades de aula ejercicios motrices de creatividad. El abrir espacios para descubrir nuevos talentos y el uso de las tecnologías fueron fundamentales en el éxito de diversos talleres. Se generó la necesidad de diseñar una página web como repositorio del trabajo de los estudiantes, a fin de dar a conocer los resultados y dar reconocimiento a los talentos detectados en el grupo.

\section{Agradecimientos}

Sinceros agradecimientos al grupo de estudiantes participantes del proceso, por su disposición en la realización y análisis de cada uno de los talleres implementados, así como a la institución objeto de estudio. 


\section{Referencias}

Alvarado, A. (2012). Estrategias para mejorar la redacción de resúmenes escritos por estudiantes de Educación Superior. Revista Universitaria de Investigación, 13(2), 15-36.

Benítez, F. M. y Rincón, Y. A. (2016). Diagnóstico del Desarrollo de las Competencias Comunicativas Orales y Escritas en Contaduría Pública. Caso Pontificia Universidad Javeriana. https://repository.javeriana. edu.co/bitstream/handle/10554/20478/BenitezGomez FannyMarcela2016.pdf? sequence $=1$ \&isAllowed $=y$

Bisquera Alzina, R. (2003). Educación emocional y competencias básicas para la vida. Revista de Investigación Educativa, 21(1), 7-43. https://revistas. um.es/rie/article/view/99071

Cassany, D. (2006a). Taller de textos: leer, escribir y comentar en el aula (13a edic.). Paidós.

Cassany, D. (2006b). Tras las líneas: sobre la lectura contemporánea. Anagrama, Barcelona.

Castaño, M. y Echenique, A. (2017). Estrategia didáctica para el desarrollo transversal de la competencia comunicativa en la formación profesional de los estudiantes de educación superior. Escenarios, 15(1), 119-130.

Fragoso-Luzuriaga, R. (2015). Inteligencia emocional y competencias emocionales en educación superior. ¿Un mismo concepto? Revista Iberoamericana de Educación Superior, 6(16), 110-125.

García Retana, J. Á. (2012). La educación emocional, su importancia en el proceso de aprendizaje. Revista Educación de La Universidad de Costa Rica, 36(1), 97109.

Goleman, D. (2010). La práctica de la inteligencia emocional. Editorial Kairós.

Icfes. (2016). Guía de orientación: Módulo de Competencias ciudadanas. https://n9.cl/ax3s

Icfes. (2017a). Resultados agregados de Saber Pro en los módulos de competencias genéricas de 2017. http://www.icfes.gov.co/instituciones-educativasy-secretarias/saber-pro/resultados-agregados/ resultados-agregados-2017

Icfes. (2017b). Saber Pro. Módulos de Competencias Genéricas.

Rincón, M. T. (2014). Formación en competencias comunicativas en educación media y su incidencia en la educaciónn superior. https://repository.usta.edu. co/handle/11634/4683

Sanchez, J. M. y Brito, N. E. (2015). Desarrollo de competencias comunicativas mediante la lectura crítica, escritura creativa y expresión oral. Encuentros, 13(2), 117-141.

Simancas, E. P. y Utria, C. A. (2013). Factores institucionales y desempeño estudiantil en las pruebas Saber-Pro de las Instituciones Públicas Técnicas y Tecnológicas del Caribe Colombiano. I+ D Revista de Investigaciones, 1(1), 60-67. https://doi.org/10.33304/revinv.v01n1-2013007
Solé, I. (2010). Estrategias de lectura. Editorial Graó.

Solé, I. (2012). Competencia lectora y aprendizaje. Revista Iberoamericana de Educación (OEI), 2012(59), 43-61. 